\title{
Apoio social, estilo parental e a saúde mental de crianças e adolescentes ${ }^{\#}$ Social support, parenting style and mental health of children and adolescents
}

\author{
Sandra Midori Sakuramoto* \\ Carolina Elisabeth Squassoni* * \\ Thelma Simões Matsukura***
}

\begin{abstract}
Resumo
A literatura aponta as práticas parentais e o apoio social recebido pelos pais como fatores que influenciam a saúde mental infanto-juvenil, sendo que as práticas podem predizer o desenvolvimento de comportamentos pró-sociais e antissociais da criança. No que diz respeito ao apoio social, verifica-se a relação entre níveis de satisfação e indicadores positivos de desenvolvimento. O objetivo do estudo foi identificar as correlações entre o apoio social dos pais, as práticas parentais familiares e a saúde mental dos filhos. Participaram 51 díades, pai ou mãe e seus filhos, com idade entre 11 e 13 anos. Utilizou-se o Questionário de apoio social, o Inventário de Estilos Parentais, o Questionário de Capacidades e Dificuldades e um questionário de identificação. As análises foram realizadas por meio do estudo de correlação de Pearson. Os resultados indicaram que quanto maior o apoio social e mais positivo o estilo parental menor é o risco de a criança apresentar algum problema em sua saúde mental. O desafio que se coloca para as pesquisas futuras e para o desenvolvimento de ações junto às famílias relaciona-se ao desenvolvimento de intervenções que possam alcançar os pais no sentido de esclarecê-los acerca da importância de práticas de cuidados e atenção cotidianas voltadas para os filhos, ainda que se considerem condições de vida adversas.
\end{abstract}

Palavras-chave: Saúde Mental. Comportamento Infantil. Fatores de Risco. Desenvolvimento Infantil. Apoio Social.

\begin{abstract}
Literature suggests that parenting practices and social support received by parents are factors that influence mental health of children and adolescents; practices may predict the development of pro-social and antisocial behavior of children; regarding social assistance, there is a relationship between satisfaction and positive indicators of development. The purpose of the study was to identify correlations between social support from parents, family parental practices and mental health of children. Fifty one pairs took part in the present study, composed by father or mother and their children with ages ranging from 11 and 13 years old. The instruments used to collect data were: Social Support Questionnaire, Parental Style Inventory, Strengths and Difficulties Questionnaire and an identification questionnaire. Data analysis was performed using Pearson's correlation study. The outcomes indicate that the greater the social support and the more positive the parental style, the less risky it is for children to present any mental health problem. The challenge for future research and development of actions among families relates to the development of interventions that can reach parents to enlighten them about the importance of practical daily care and attention to children, even when there are adverse living conditions.
\end{abstract}

Keywords: Mental Health. Child Behavior. Risk Factors. Child Development. Social Support.

DOI: $10.15343 / 0104-7809.20143802169178$

\# Artigo derivado de: "Sakuramoto SM. Identificando Fatores protetivos ao desenvolvimento em crianças em situação de risco pessoal e social [Relatório de Iniciação Científica]. São Carlos (SP): Universidade Federal de São Carlos; 2008. (Centro de Ciências Biológicas e da Saúde, Departamento de Terapia Ocupacional)".

* Centro de Atenção Integrada em Saúde Mental - Hospital da Água Funda (CAISM), São Paulo-SP, Brasil.

** Departamento de Terapia Ocupacional da Universidade Federal de São Carlos, São Carlos-SP, Brasil.

*** Departamento de Terapia Ocupacional da Universidade Federal de São Carlos, São Carlos-SP, Brasil. E-mail: thelma@ufscar.br As autoras declaram não haver conflitos de interesse. 


\section{INTRODUC̣ÃO}

Muitas crianças e adolescentes crescem e se desenvolvem em contextos e situações que constituem ameaças à sua saúde física e psicossocial. Ao enfrentar situações de estresse e risco no seu cotidiano, elas podem apresentar distúrbios emocionais e problemas de conduta ${ }^{1}$. Os fatores de risco estão relacionados a todo tipo de eventos negativos de vida e que, quando presentes, aumentam a probabilidade de o indivíduo apresentar problemas físicos, sociais ou emocionais.

A literatura aponta inúmeros fatores considerados de risco para crianças e adolescentes. São eles: baixo nível socioeconômico; condição de miserabilidade; grande número de indivíduos na composição da família nuclear; ocupação de baixo status dos pais; divórcio dos pais; perda de emprego pelos pais; morte de familiares próximos; ausência de um dos pais; baixa escolaridade (tanto do adolescente como dos pais); conflitos familiares; violência psicológica, física ou sexual perpetrada contra a criança ou qualquer outro membro da família; nível de estresse da família; baixa expectativa dos pais quanto ao desenvolvimento dos filhos; práticas educativas ineficazes; psicopatologia na família e presença de deficiência física ${ }^{2,3}$.

De acordo com Gaspar, Matos e Ribeiro ${ }^{4}$, a pobreza tem sido descrita como uma condição especialmente geradora de estresse e sofrimento dentro do panorama multidimensional do nível socioeconômico, pois isso significa a exposição a situações que provocam uma deterioração, ou seja, estar sujeito a condições desfavoráveis acarreta em um grande risco para a saúde e bem-estar físico, mental e social da criança e adolescente, podendo também afetar a estabilidade e o bom desenvolvimento das relações familiares.

A presença de adversidades econômicas influencia no aumento de situações estressantes nas famílias e nos fatores de risco para o desenvolvimento de condutas parentais inadequadas, incluindo maus tratos, negligência e abandono ${ }^{5}$.

Salvador e Weber ${ }^{6}$ apontam que a figura dos pais, como primeiro núcleo social da criança, tem grande influência em seu processo de desenvolvimento social, cognitivo e psicológico. A relação entre pais e filhos pode ser estudada por meio das práticas educativas parentais. As práticas parentais correspondem a estratégias utilizadas pelos pais para orientar o comportamento dos filhos?.

Vários autores vêm estudando as práticas educativas parentais nas últimas décadas, observando as possíveis implicações das práticas educativas utilizadas pelos pais no desenvolvimento de seus filhos ${ }^{8-10}$.

Santos e Graminha ${ }^{11}$, em uma revisão da literatura, verificaram que famílias de alto risco, cujos filhos obtiveram sucesso em termos cognitivos, tinham maior clareza na colocação e explicação de regras, eram menos democráticas e justificavam menos sua política educacional do que as famílias de baixo risco. Tais resultados sugerem que as práticas educativas podem se apresentar como fatores de proteção para crianças de famílias de alto risco.

Salvo, Silvares e Toni ${ }^{12}$, no estudo realizado com 30 estudantes de 11 a 13 anos e seus pais, concluíram que determinadas práticas educativas podem predizer o comportamento da criança, com um posterior desenvolvimento de comportamentos pró-sociais e antissociais nas mesmas. A ausência da prática de monitoria positiva foi associada ao comportamento agressivo da criança, e a sua presença foi associada com a sociabilidade; o comportamento moral foi relacionado à competência social, e a monitoria negativa se mostrou relevante para os problemas de atenção e de internalização da criança.

A literatura aponta que o desenvolvimento de estudos a respeito das relações entre práticas educativas parentais e o desenvolvimento psicológico e social das crianças podem contribuir para o planejamento de estratégias de intervenção que possibilitem a prevenção e tratamento de problemas de comportamento em crianças $^{8,10}$.

Assim como as práticas parentais, o apoio social recebido pelos pais também é apontado como importante aspecto que influencia na saúde mental infanto-juvenil, no sentido de quanto maior o apoio, melhor os indicadores de desenvolvimento da criança ${ }^{13-15}$.

$\mathrm{Na}$ concepção de $\mathrm{Vaux}^{16}$, o apoio social refere-se ao apoio emocional, prático e material dado pela família, amigos e pessoas próximas a um indivíduo, na forma de afeto, companhia, 
assistência e informação, fazendo com que se sinta amado, estimado, cuidado, valorizado, encorajado e pertencente a um grupo. Ainda de acordo com o autor, o apoio social deve ser considerado como um metaconstruto com três componentes conceituais distintos, ou seja, recursos da rede de apoio, comportamento de apoio (incluem-se aqui os diferentes tipos de apoio) e as avaliações subjetivas do apoio social.

De acordo Burchinal, Follmer e Briant ${ }^{13}$, o tamanho da rede de apoio social da mãe pode influenciar no sistema social da família, facilitando o estilo parental positivo da mãe. Mães com maior rede de apoio social tendem a dar mais assistência a seus filhos e um maior número de interações diárias quando em comparação com as mães com menor rede de apoio; além disso, tendem a ser mais responsivas, abertas, envolvidas com seus filhos e menos controladoras do comportamento da criança.

O estudo de Ceballo e McLoyd ${ }^{17}$ verificou a relação entre apoio social e estratégias de parenting de mães americanas que viviam em uma comunidade socialmente desfavorecida. A pesquisa foi conduzida a partir de entrevistas com 282 mães americanas afrodescendentes solteiras e suas crianças que viviam em locais cujas vizinhanças eram consideradas perigosas - enquanto condição de estresse ambiental. Os resultados indicaram que as condições da vizinhança atuam como moderadoras na relação entre apoio social e parenting, sendo que, quando as condições da vizinhança são melhores, a relação negativa encontrada entre apoio social recebido e comportamentos parentais punitivos ficou mais fortalecida. As autoras concluem que o apoio social influencia de forma positiva o parenting, além de atenuar os efeitos da pobreza e da vizinhança ameaçadora.

De acordo com o exposto, espera-se que o presente estudo possa contribuir para o aumento da compreensão e do conhecimento nessa área, a partir de reflexões e proposições de práticas e políticas públicas que busquem minimizar e/ou prevenir problemas de comportamento e saúde mental em crianças e adolescentes expostas a situações de risco.

Considerando as informações apresentadas, o objetivo do presente estudo foi identificar correlações entre o apoio social dos pais, práticas parentais familiares e saúde mental dos filhos.

\section{MÉTODO}

\section{Participantes}

Participaram deste estudo 51 díades criança e mãe (ou pai). Como critérios de inclusão, as crianças e adolescentes deveriam ter idade entre 11 e 18 anos e pertencerem a famílias intactas, ou seja, morarem em um mesmo local com seus pais e mães não separados.

O levantamento dos participantes foi realizado com base no cadastro de todas as crianças e adolescentes rematriculadas e/ou matriculadas em instituições não governamentais de atendimento a criança e adolescente, em situação de risco pessoal e social, de uma cidade do interior do estado de São Paulo. No total das 607 matrículas analisadas, apenas 81 responderam aos critérios do estudo e 51 famílias aceitaram participar. Na Tabela 1, apresenta-se o perfil das crianças e dos adolescentes participantes.

Tabela 1. Perfil das crianças e adolescentes segundo sexo, idade e série

\begin{tabular}{|c|c|c|c|c|c|c|c|c|}
\hline \multirow{2}{*}{ Sexo } & \multirow{2}{*}{ Idade } & \multicolumn{6}{|c|}{ Série } & \multirow{2}{*}{ Total gera } \\
\hline & & $3^{a}$ & $4^{a}$ & $5^{\mathrm{a}}$ & $6^{a}$ & $7^{a}$ & $8^{a}$ & \\
\hline \multirow{3}{*}{ Feminino } & 11 & 1 & 1 & 12 & 1 & 0 & 0 & 15 \\
\hline & 12 & 0 & 1 & 1 & 2 & 1 & 0 & 5 \\
\hline & 13 & 0 & 0 & 0 & 0 & 6 & 1 & 7 \\
\hline \multirow{3}{*}{ Masculino } & 11 & 0 & 5 & 3 & 3 & 0 & 0 & 11 \\
\hline & 12 & 0 & 0 & 0 & 4 & 0 & 0 & 4 \\
\hline & 13 & 0 & 0 & 2 & 2 & 5 & 0 & 9 \\
\hline Total geral & & 1 & 7 & 18 & 12 & 12 & 1 & 51 \\
\hline
\end{tabular}


Verifica-se na Tabela 1 que a idade dos participantes variou de 11 a 13 anos, com predominância de alunos da $5^{\text {a }}$ série do ensino fundamental. Com relação ao sexo, 27 eram meninas. No que diz respeito ao perfil dos pais e mães respondentes verificou-se que destes, 46 eram mães e cinco eram pais. Com relação à idade, a maioria dos pais $(n=30)$ e mães $(n=32)$ encontrava-se na faixa etária entre 30 e 40 anos.

Os dados referentes à escolaridade apontaram que 25 pais e 20 mães possuíam até a $4^{\text {a }}$ série do ensino fundamental, 16 mães e 6 pais possuíam o ensino médio completo, 5 pais e 3 mães não foram alfabetizados.

\section{Local}

O estudo foi realizado em cinco instituições não governamentais de atendimento à criança e ao adolescente, em situação de risco pessoal e social. O risco relacionava-se ao baixo nível socioeconômico de suas famílias. Todas as instituições estavam cadastradas no Conselho Municipal de Direitos da Criança e do Adolescente e situadas em uma cidade do interior do estado de São Paulo. As instituições oferecem programas de atividades em período oposto ao escolar para crianças e adolescentes estudantes da rede pública de ensino, de segunda a sexta-feira.

A coleta de dados com as crianças e adolescentes ocorreu em suas instituições de vínculo. Já a coleta com os pais ocorreu em horários e locais estabelecidos conforme opção da família.

\section{Instrumentos}

\section{Questionário de Apoio social}

O Questionário de Apoio social - SSQ ${ }^{18}$ foi aplicado junto ao pai ou à mãe da criança e do adolescente com objetivo de medir o apoio social dos mesmos. Foi utilizada a versão brasileira do SSQ cujos resultados do estudo de adaptação e qualidades psicométricas indicaram uma fidedignidade teste-reteste aceitável e um alto nível de consistência interna, sendo que os coeficientes alpha de Cronbach obtidos foram, para a escala N (SSQ-N), 0,9384 no teste e de 0,9610 no reteste. Para a Escala S (SSQ-S) obteve-se 0,9438 no teste e 0,9626 no reteste ${ }^{18}$.
O SSQ é constituído por 27 questões relacionadas a situações problemáticas do cotidiano nas quais, em sua primeira parte, o respondente indica o nome das pessoas que podem lhe prestar apoio em tal situação e o tipo de relacionamento com essa pessoa (ex: amigo, irmã, marido). Na segunda parte das questões, o respondente deve indicar seu grau de satisfação com esse apoio ${ }^{18}$.

Sendo assim, o SSQ fornece dois escores: o SSQ-N e o SSQ-S. O SSQ-N é o índice calculado a partir da divisão entre o total de pessoas citadas em todas as questões do instrumento pelo número de questões (27). O índice SSQ-S informa sobre a satisfação do indivíduo com o apoio recebido, sendo que o valor poderia variar de 1 a 6 . Além disso, a alternativa "muito satisfeito" equivale a 6 pontos e "muito insatisfeito" a 1 ponto ${ }^{18}$.

\section{Inventário de Estilos Parentais (IEP)}

O IEP ${ }^{19}$ contém 42 questões abordando práticas educativas positivas e negativas e pode ser respondido tanto pelos pais (que respondem sobre como utilizam as práticas educativas com seus filhos), quanto pelas crianças e adolescentes. No presente estudo, o instrumento foi respondido pelos pais e seus filhos. $\mathrm{O}$ instrumento foi desenvolvido a partir de práticas educativas selecionadas pela autora, a saber: monitoria positiva, comportamento moral, negligência, abuso físico, abuso psicológico, disciplina relaxada, punição inconsistente e monitoria negativa ${ }^{19}$.

O IEP apresenta estudos de validação no Brasil, sendo que os resultados para avaliar o estilo parental materno revelaram coeficientes razoáveis de consistência interna, variando de 0,47 (monitoria negativa) a 0,82 (abuso físico) ${ }^{20}$.

A tabulação de dados foi feita utilizando a folha de resposta que contém as sete práticas educativas do instrumento. Cada resposta "nunca" recebeu a pontuação 0; "às vezes" pontuação 1 ; "sempre" pontuação 2. Desse modo, cada prática educativa pode ter a pontuação máxima de 12 e a mínima de 0 . O índice de estilo parental foi calculado subtraindo a soma das práticas negativas (punição inconsistente; negligência; disciplina relaxada; monitoria negativa e abuso físico) da soma das práticas positivas (monitoria positiva 
e comportamento moral). Assim, quando o resultado for negativo, o estilo parental será negativo; se o resultado for positivo, o estilo parental será positivo. Os escores revelam o estilo parental adotado pelos pais: estilo parental ótimo; regular acima da média; regular abaixo da média e de risco ${ }^{20}$.

\section{Questionário de Capacidades e Dificuldades (SDQ)}

O SDQ ${ }^{21}$ foi utilizado para avaliar a saúde mental das crianças e adolescentes e foi respondido pelos pais e seus filhos. É composto por 25 itens, subdivididos em 5 subescalas que avaliam os seguintes itens: hiperatividade, sintomas emocionais, problemas de conduta, relações interpessoais e comportamento prossocial.

No Brasil, os estudos de validação do SDQ foram realizados por Fleitlich-Bilyk ${ }^{22}$ a partir da validade de critério preditiva, na qual houve comparação entre os resultados obtidos com as três versões do SDQ (criança, pai, professor) com uma avaliação psiquiátrica independente. O estudo foi realizado com uma amostra aleatória de crianças identificadas com transtorno psiquiátriCO, e os resultados do SDQ se relacionaram com os resultados da avaliação psiquiátrica.

Os resultados do SDQ são obtidos a partir da pontuação de cinco escalas primeiramente e, em seguida, calcula-se o total de dificuldades pela soma dos resultados de todas as escalas, exceto a escala de sociabilidade, cujo escore indica a capacidade da criança e do adolescente com relação ao comportamento prossocial. A opção "mais ou menos verdadeiro" vale 1 ponto e as alternativas "falso" e "verdadeiro" é calculada conforme o item, podendo valer 0 ou $2^{23}$.

As possibilidades de resultados indicados pelo instrumento para todas as cinco subescalas e para o total de dificuldades são três: "Normal" (saudável): indica que não há dificuldades relativas ao que está sendo avaliado; "Limítrofe": indica que a criança ou o adolescente já apresenta alguma dificuldade que, se não for devidamente cuidada, pode se agravar e prejudicar seu desenvolvimento; "Anormal" (clínico): indica que há dificuldades importantes relativas ao que está sendo avaliado, necessitando de intervenção especializada ${ }^{23}$.

\section{Questionário de Identificação e caracterização da criança e do adolescente}

Para a caracterização da realidade familiar e social da criança e do adolescente, foi utilizado um questionário elaborado pelas pesquisadoras que foi respondido pelos pais. Esse questionário abordou aspectos como a composição familiar, as condições de moradia, a rotina diária e o desempenho escolar das crianças.

\section{Procedimentos}

A presente pesquisa foi submetida ao Comitê de Ética em Pesquisa da Universidade Federal de São Carlos e obteve aprovação sob parecer n. 85/2008. Após a assinatura do Termo de Consentimento Livre e Esclarecido, iniciaram-se os procedimentos para a coleta de dados.

\section{Análise dos dados}

A fim de analisar as possíveis correlações existentes entre as escalas SSQ e IEP, SSQ e SDQ e entre o SDQ e IEP, foi utilizado o Coeficiente de Correlação de Pearson. Com o objetivo de garantir um nível seguro de significância, foi considerado p-valores menores que 0,05. Além disso, foram realizados testes de normalidade, por meio da Análise de Resíduos, a fim de garantir a validade das análises.

\section{RESULTADOS}

Inicialmente, serão apresentados os resultados descritivos referentes aos instrumentos: SSQ, Inventário de Estilos Parentais e Questionário de Capacidades e Dificuldades, respectivamente. Em seguida, serão apresentados os resultados obtidos a partir das correlações.

\section{SSO}

No que se refere à fonte de apoio, a família extensa (pais, irmãos, tios, primos, cunhados) foi indicada como a maior fonte de suporte presente em $43,77 \%$ da possibilidade de fontes, seguida pela família próxima (filhos e o cônjuge), com 42,89\%.

Quanto ao número de fontes de apoio, a média de fontes de suporte entre os pais 
respondentes foi de 2,16 (poderiam citar até nove possibilidades ou a opção "ninguém").

Com relação à satisfação com o apoio recebido, a satisfação foi alta para a maioria dos respondentes, sendo 5,4 a média dos escores. Apenas quatro não estavam satisfeitos com o apoio, obtendo escores abaixo de 4.

\section{Inventário de Estilos Parentais}

De acordo com os resultados obtidos a partir do Inventário de Estilos Parentais, verificou-se na versão respondida pelos pais: estilo parental ótimo (19 pais), regular acima da média (9 pais), regular abaixo da média (13 pais) e estilo parental de risco (10 pais).

$\mathrm{Na}$ versão respondida pelas crianças e adolescentes, classificou-se o estilo parental como ótimo $(n=2)$, regular acima da média $(n=15)$, regular abaixo da média $(n=12)$ e estilo parental de risco $(n=22)$. Os resultados também demonstraram que os pais avaliaram suas práticas paren- tais melhor do que seus filhos o fizeram, pois a média de estilo parental dos pais foi "regular acima da média", enquanto a dos filhos foi "regular abaixo da média".

Comparando-se o índice de estilo parental dos pais com o de seus respectivos filhos, observou-se que apenas seis díades pai-filho apresentaram o mesmo estilo parental, enquanto que a maioria dos filhos percebeu as práticas parentais como mais negativas do que seus pais. Apenas nove crianças avaliaram as práticas de forma mais positivas do que seus pais.

\section{Questionário de Capacidades e Dificulda-} des

Na Tabela 2, são apresentados os resultados obtidos por meio do instrumento SDQ, no qual são verificadas as respostas dos pais referentes à saúde mental de seus filhos e a resposta das crianças sobre elas mesmas.

Tabela 2. Classificação do SDQ respondido pelas crianças e pelos pais

\begin{tabular}{lcccccc}
\hline Classificação & \multicolumn{2}{c}{ Saudável(n) } & \multicolumn{2}{c}{ Limítrofe(n) } & \multicolumn{2}{c}{ Clínico(n) } \\
\hline Respondente & criança & pai & criança & pai & criança & pai \\
\hline Escala de sintomas emocionais & $(33)$ & $(17)$ & $(6)$ & $(6)$ & $(12)$ & $(28)$ \\
Escala de problemas de conduta & $(27)$ & $(34)$ & $(10)$ & $(5)$ & $(14)$ & $(12)$ \\
Escala de hiperatividade & $(40)$ & $(33)$ & $(3)$ & $(3)$ & $(8)$ & $(15)$ \\
Escala de problemas de relacionamento & $(29)$ & $(26)$ & $(16)$ & $(10)$ & $(6)$ & $(15)$ \\
Escala de comportamento prossocial & $(43)$ & $(49)$ & $(3)$ & $(1)$ & $(6)$ & $(1)$ \\
Total de dificuldades & $(30)$ & $(26)$ & $(8)$ & $(6)$ & $(13)$ & $(19)$ \\
Suplemento de impacto & $(39)$ & $(33)$ & $(3)$ & $(5)$ & $(9)$ & $(13)$ \\
\hline
\end{tabular}

De acordo com os resultados verificados na Tabela 2, observa-se que os pais apontaram que seus filhos possuem mais dificuldades relacionadas à sua saúde mental do que eles próprios se avaliaram. Ainda de acordo com os resultados do SDQ, observa-se que 19 pais avaliaram seus filhos com um total de dificuldades considerado clínico. No que se refere aos casos clínicos, 28 pais consideraram que seus filhos apresentam sintomas emocionais.

Com relação à versão de autoavaliação, verifica-se que 13 crianças se avaliaram com sintomas de dificuldades clínicas, com predominância de problemas de conduta e sintomas emocionais.

\section{Resultados das correlações}

Correlação entre apoio social dos pais e práticas parentais familiares

O estilo parental respondido pelos pais foi correlacionado positivamente com o apoio social, tanto com sua satisfação, como com o número de pessoas que fornecem apoio. Isso indica que quanto maior o apoio social dos pais, maior o uso de práticas parentais positivas. Tais correlações são apresentadas na Tabela 3. 
Tabela 3. Resultados do teste de Correlação de Pearson: IEP e SSQ-S e IEP e SSQ-N

\begin{tabular}{lccccc}
\hline & & \multicolumn{2}{c}{ SSQ-S } & \multicolumn{2}{c}{ SSQ-N } \\
\hline & $\mathrm{n}$ & $\mathrm{r}$ & $\mathrm{p}$-valor & $\mathrm{r}$ & $\mathrm{p}$-valor \\
IEP-pai & 51 & 0,31 & $\mathbf{0 , 0 3}$ & 0,34 & $\mathbf{0 , 0 2}$ \\
IEP-filho & 51 & 0,19 & 0,18 & 0,00 & 0,98 \\
\hline
\end{tabular}

p-valor $<0,05$.

\section{Correlação entre apoio social dos pais $e$ saúde mental das crianças e adolescentes}

Analisando os resultados da correlação entre as dificuldades da criança e adolescentes e o apoio social recebido pelos pais, identificou-se a correlação negativa entre a satisfação com o apoio social e a escala do SDQ de problemas de relacionamento respondido pelos pais, ou seja, quanto maior a satisfação dos familiares com o apoio social recebido, menor a chance de o filho apresentar problemas de relacionamento com os colegas e vice-versa. Essa correlação foi verificada na Tabela 4.

Tabela 4. Resultados do teste de Correlação de Pearson - subescalas do SDQ (versão para pais) e SSQ-S $(\mathrm{n}=51)$

\begin{tabular}{lcc}
\hline SDQ & $\mathbf{r}$ & p-valor \\
\hline Sintomas emocionais & $-0,15$ & 0,31 \\
Problemas de conduta & $-0,04$ & 0,77 \\
Hiperatividade & 0,06 & 0,65 \\
Problemas de relaciona- & $-0,36$ & 0,01 \\
mento & & \\
Comportamento prossocial & 0,15 & 0,30 \\
Total de dificuldades & $-0,13$ & 0,35 \\
Suplemento de impacto & $-0,20$ & 0,151 \\
\hline
\end{tabular}

p-valor $<0,05$.

\section{Correlação entre práticas parentais familia- res e saúde mental das crianças e adolescentes}

O índice de estilo parental avaliado pelos pais foi significativamente correlacionado com o SDQ respondido pelos pais em várias subescalas, sendo que este teve correlação negativa com o total de dificuldades da criança e adolescente, na escala de problemas de conduta e hiperatividade, e correlação positiva com o comportamento prossocial, como se observa na Tabela 5.
Tabela 5. Resultados do teste de Correlação de Pearson - subescalas do SDQ (versão para pais) e IEP (versão para pais) $(n=51)$

\begin{tabular}{lcc}
\hline \multirow{2}{*}{ SDQ } & \multicolumn{2}{c}{ IEP } \\
\hline Sintomas emocionais & $r$ & p-valor \\
Problemas de conduta & $-0,27$ & 0,06 \\
Hiperatividade & $-0,47$ & $\mathbf{0 , 0 0 0 4}$ \\
Problemas de relacionamento & $-0,35$ & $\mathbf{0 , 0 1}$ \\
Comportamento prossocial & 0,45 & $\mathbf{0 , 0 0 1}$ \\
Total de dificuldades & $-0,48$ & $\mathbf{0 , 0 0 0 4}$ \\
Suplemento de impacto & 0,00 & 0,98 \\
\hline
\end{tabular}

p-valor $<0,05$.

Quando os filhos foram os respondentes do SDQ, encontrou-se correlação negativa entre o IEP da criança e adolescente e a escala de problemas de relacionamento com os colegas. Assim, quanto melhor o estilo parental adotado pelo pai seja esse avaliado pelo próprio ou pela criança, menor os problemas de saúde mental apresentados pelo filho.

\section{DISCUSSÃO}

No presente estudo os resultados indicaram que o apoio social dos pais foi correlacionado com a saúde mental dos filhos. Tal resultado apresenta concordância com outros estudos, entre eles o de Demaray, et $\mathrm{a}^{14}$, que aponta a relação entre apoio social recebido pelos pais e desenvolvimento saudável dos filhos. Mota, Franco e Motta ${ }^{15}$ apontam que o grau de satisfação materna com o apoio social desempenha o papel central no desenvolvimento emocional infantil, pois isso contribui para moderar o efeito negativo de uma situação estressante, protegendo as crianças contra a incerteza, cuidados precários, violência e isolamento.

Importa destacar que no presente estudo foi a satisfação com o apoio social recebido que esteve relacionada à saúde mental da criança e não o número de pessoas suportivas. Assim, compreende-se que, mais que a quantidade de pessoas disponíveis para o fornecimento de apoio social aos pais, importa a satisfação com que esses cuidadores compreendem o apoio recebido dessas pessoas. 
Não obstante, este estudo identificou que as práticas parentais positivas estão relacionadas à saúde mental da criança e do adolescente e que o apoio social se relaciona a tais práticas parentais. Nesse caso, tanto a satisfação quanto a quantidade do apoio social recebido estão relacionadas às práticas parentais adequadas. Nesse sentido, tanto quantidade e quanto qualidade do apoio parecem interferir no processo - diretamente em relação às práticas parentais e, indiretamente, em relação à saúde mental da criança e do adolescente.

O achado acerca da associação entre apoio social e estilo parental confirma resultados de estudos anteriores ${ }^{9,13}$. Mães com maior rede de apoio social tendem a dar mais assistência a seus filhos e um maior número de interações por dia comparado às mães com menor rede apoio social.

Em relação às práticas parentais e saúde mental das crianças, este estudo encontrou correlação negativa entre as práticas parentais negativas e a saúde mental da criança (total de dificuldades da criança, problemas de conduta e hiperatividade), e correlação positiva com o comportamento prossocial. Tal resultado corrobora o estudo de Gomide, et $\mathrm{al}^{24}$, no qual foram verificadas associações entre práticas educativas deficitárias, depressão, estresse e habilidades sociais, cujos resultados demonstraram correlação positiva entre o IEP positivo e o alto índice de habilidades sociais. Por outro lado, as famílias com IEP negativo apontaram insuficiente repertório de habilidades sociais.

Os resultados do presente estudo revelaram que os filhos avaliaram as práticas parentais de seus pais de forma mais negativa que os seus próprios pais quando responderam as mesmas questões. Aponta-se que estudos futuros poderiam focalizar onde essas discrepâncias aparecem de forma consistente e quais fatores podem estar presentes nessa defasagem. Hipotetiza-se que os pais possam responder buscando revelar comportamentos socialmente esperados e aceitos, não obstante, o instrumento parece sensível para detecção de diferentes estilos parentais. De qualquer forma, esta pesquisa aponta para a adequação de que ambos, pais e filhos, respondam ao instrumento nos estudos que utilizem o IEP.
Na mesma direção, aponta-se consideração semelhante em relação ao uso do SDQ.

Ainda no que tange às práticas parentais, julga-se necessária a articulação de uma rede de auxílio às famílias para lidarem com as dificuldades encontradas na educação dos filhos; auxiliá-las na estruturação, organização e enriquecimento do ambiente de desenvolvimento das crianças; possibilitar o desenvolvimento de práticas educativas mais positivas e eficazes, a fim de promover desenvolvimento das crianças ${ }^{25}$.

Nessa direção, conforme aponta $\mathrm{Cid}^{26}$, tanto os fatores de risco como os fatores de proteção influenciam de forma significativa a saúde mental do indivíduo no que diz respeito ao desenvolvimento de comportamentos de risco, desenvolvimento emocional e também no desenvolvimento cognitivo. Para a autora, identificar esses problemas e suas causas é fundamental para que estratégias de intervenção direcionadas à prevenção e/ou promoção da saúde mental de crianças sejam planejadas.

De toda forma, aponta-se para a necessidade de se compreender a saúde mental infantil a partir de um contexto mais amplo, no qual os mecanismos de risco vivenciados pelas famílias sejam considerados nas ações de promoção, prevenção e tratamento em saúde mental, a partir de ações intersetoriais, por meio da articulação entre os diferentes setores públicos que assistem crianças e adolescentes ${ }^{26}$

\section{CONCLUSÃO}

Ainda que com os limites de um número reduzido de participantes, o que não permite generalizações, e considerando também que o presente estudo envolveu apenas famílias intactas, o que representa apenas parte da realidade das famílias brasileiras, o presente estudo identificou possíveis correlações entre o apoio social dos pais, as práticas parentais familiares e a saúde mental de crianças e adolescentes em situação de risco pessoal e social. Com este estudo, aponta-se para a importância dos pais como educadores e no processo de desenvolvimento dos filhos, que é influenciado por diversos fatores, sendo a família um dos mais importantes. O desafio que se coloca para pesquisas futuras e para o desenvolvimento 
de ações junto às famílias relaciona-se ao desenvolvimento de intervenções que possam alcançar pais no sentido de sensibilizá-los ou esclarecê-los sobre a importância de práticas de cuidados e atenção cotidianas voltadas para os filhos, ainda que se considerem condições de vida bastante adversas.

Assim, ressalta-se a importância de políticas públicas e de intervenção direcionadas a essa população, destacando-se também o papel do apoio social como importante fator protetivo no processo de desenvolvimento humano.
Conclui-se que o presente estudo colaborou para a compreensão da realidade de famílias, principalmente no que se refere à população menos favorecida, ampliando o entendimento sobre as relações familiares e a consequente implicação delas no desenvolvimento da criança e do adolescente.

\section{AGRADECIMENTOS}

A todos que colaboraram para a realização deste estudo, em especial aos participantes, pais e seus filhos.

\section{REFERÊNCIAS}

1. Cecconello AM, Koller SH. Competência social e empatia: um estudo sobre resiliência com crianças em situação de pobreza. Estud Psicol. 2000 [acesso 10 Ago 2013];5(1):71-93. Disponível em: http://www.scielo.br/scielo. php?script=sci arttext\&pid=S1413-294X2000000100005\&lng=pt\&nrm=iso\&tlng=pt. DOI: http://dx.doi.org/10.1590/ s1413-294x2000000100005.

2. Hutz CS. Violência e risco na infância e adolescência: pesquisa e intervenção. São Paulo: Casa do Psicólogo; 2005.

3. Bardagi MP, Arteche AX, Neiva-Silva L. Projetos sociais com adolescentes em situação de risco: discutindo o trabalho e a orientação profissional como estratégias de intervenção. In: Hutz CS, editor. Violência e risco na infância e na adolescência: pesquisa e intervenção. São Paulo: Casa do Psicólogo; 2005. p. 101-46.

4. Gaspar T, Matos MG, Ribeiro JLP, Leal I. Qualidade de vida e bem-estar em crianças e adolescentes. Rev Bras Ter Cogn. 2006;2(2):47-60.

5. Bringiotti MI, Krynveniuk M, Lasso S. Las multiples violencias de la "violencia" en la escuela. Desarrollo de un enfoque teorico y metodologico integrativo. Paideia. 2004 [acceso 20 Jun 2013];14(29):313-25. Disponible en: http://www. scielo.br/scielo.php?script=sci arttext\&pid=S0103-863X2004000300007\&lng=es\&nrm=iso\&tlng=es. DOI: $\underline{\text { http://dx.doi }}$. org/10.1590/s0103-863x2004000300007.

6. Salvador APV, Weber LND. Práticas educativas parentais: um estudo comparativo da interação familiar de dois adolescentes distintos. Interação Psicologia. 2005;9(2):341-53.

7. Reppold CT, Pacheco J, Bardagi M, Hutz CS. Prevenção de problemas de comportamento e desenvolvimento de competências psicossociais em crianças e adolescentes: uma análise das práticas educativas e dos estilos parentais. In: Hutz SC, organizador. Situações de risco e vulnerabilidade na infância e na adolescência: aspectos teóricos e estratégia de intervenção. São Paulo: Casa do Psicólogo; 2002. p. 7-52.

8. Alvarenga P, Piccinini C. Práticas educativas maternas e problemas de comportamento em pré-escolares. Psicol Reflex Crit. 2001 [acesso 17 Jul 2013];14(3):449-69. Disponível em: http://www.scielo.br/scielo.php?script=sci arttext\&pid=S0102-79722001000300002\&lng=pt\&nrm=iso\&tlng=pt. DOI: http://dx.doi.org/10.1590/s0102-79722001000300002.

9. Leinonen JA, Solantaus TS Punamäki RL. Social support and the quality of parenting under economic pressure and workload in Finland: the role of family structure and parental gender. J Family Psychology. 2003;17(3):409-18. DOI: http:// dx.doi.org/10.1037/0893-3200.17.3.409.

10. Sapienza G, Pedromônico MRM. Risco, proteção e resiliência no desenvolvimento da criança e do adolescente. Psicol Estud. 2005 [acesso 19 Jul 2013];10(2):209-16. Disponível em: http://www.scielo.br/scielo.php?script=sci arttext\&pid=S1413-73722005000200007\&lng=pt\&nrm=iso\&tlng=pt. $\quad$ DOI: $\underline{\text { http://dx.doi.org/10.1590/s1413- }}$ 73722005000200007.

11. Santos PL, Graminha SSV. Estudo comparativo das características do ambiente familiar de crianças com alto e baixo rendimento acadêmico. Paideia. 2005 [acesso 22 Jun 2013];15(31):217-26. Disponível em: http://www.scielo.br/scielo.php?script=sci arttext\&pid=S0103-863X2005000200009\&lng=pt\&nrm=iso\&tlng=pt. DOI: $\underline{\text { http://dx.doi.org/10.1590/ }}$ $\underline{\text { s0103-863x2005000200009. }}$.

12. Salvo CG, Silvares EFM, Toni PM. Práticas educativas como forma de predição de problemas de comportamento e competência social. Estud Psicol. 2005 [acesso 7 Ago 2013];22(2):187-95. Disponível em: http://www.scielo.br/scielo. php?script=sci arttext\&pid=S0103-166X2005000200008\&lng=pt\&nrm=iso\&tlng=pt. DOI: $\underline{\text { http://dx.doi.org/10.1590/ }}$ $\underline{\text { s0103-166x2005000200008. }}$. 
13. Burchinal MR, Follmer A, Briant DM. The relations of maternal social support and family structure with maternal responsiveness and child outcomes among african american families. Dev Psychol. 1996;32(6):1073-83. DOI: http://dx.doi. org/10.1037/0012-1649.32.6.1073.

14. Demaray MK, Malecki CK, Davidson LM, Hodgson KK, Rebus PJ. The relationship between social support and student adjustment: a longitudinal analysis. Psychol Sch. 2005;42(7):691-706. DOI: http://dx.doi.org/10.1002/pits.20120.

15. Mota ELA, Franco ALS, Motta MC. Migração, estresse e fatores psicossociais na determinação da saúde da criança. Psicol Reflex Crit. 1999 [acesso 27 Jul 2013];12(1):119-32. Disponível em: http://www.scielo.br/scielo.php?script=sci arttext\&pid=S0102-79721999000100008\&Ing=pt\&nrm=iso\&tlng=pt. $\quad$ DOI: $\quad$ http://dx.doi.org/10.1590/s010279721999000100008.

178 DOI: http://dx.doi.org/10.1177/0265407590074007.

17. Ceballo R, McLoyd VC. Social support and parenting in poor, dangerous neighborhoods. Child Dev. 2002;73(4):131021.

18. Matsukura TS, Marturano EM, Oishi J. O Questionário de Suporte Social (SSQ): estudos da adaptação para o português. Rev Lat Am Enfermagem. 2002 [acesso 23 Jun 2013];10(5):675-81. Disponível em: http://www.scielo.br/scielo.php?script=sci arttext\&pid=S0104-11692002000500008\&lng=pt\&nrm=iso\&tlng=pt. DOI: http://dx.doi.org/10.1590/ s0104-11692002000500008.

19. Gomide PIC. Estilos parentais e comportamento anti-social. In: Del Prette A, Del Prette ZAP, organizadores. Habilidades sociais, desenvolvimento e aprendizagem: questões conceituais, avaliação e intervenção. Campinas (SP): Alínea; 2003. p. 21-60.

20. Gomide PIC. Inventário de Estilos Parentais (IEP): modelo teórico - manual de aplicação, apuração e interpretação. Petrópolis (RJ): Vozes; 2006.

21. Fleitlich B, Cortazar PG, Goodman R. Questionário de Capacidades e Dificuldades (SDQ). Rev Infanto Neuropsiquiatr Infânc Adolesc. 2000;8(1):44-50.

22. Fleitlich-Bilyk BW. The prevalence of psychiatric disorders in 7-14-year olds in the southeast of Brazil [thesis]. London: London University; 2002.

23. Cury CR, Golfeto JH. Strengths and difficulties questionnaire (SDQ): a study of school children in Ribeirão Preto. Rev Bras Psiquiatr. 2003 [cited 2013 Jul 7];25(3):139-45. Disponível em: http://www.scielo.br/scielo.php?script=sci arttext\&pid=S1516-44462003000300005\&lng=en\&nrm=iso\&tlng=en. $\quad$ DOI: $\underline{\text { http://dx.doi.org/10.1590/s1516- }}$ 44462003000300005.

24. Gomide PIC, Salvo CG, Pinheiro DPN, Sabbag GM. Correlação entre práticas educativas, depressão, estresse e habilidades sociais. PsicoUSF. 2005 [acesso 15 Ago 2013];10(2):169-78. Disponível em: http://www.scielo.br/scielo. php?script=sci arttext\&pid=S1413-82712005000200008\&lng=pt\&nrm=iso\&tlng=pt. $\quad$ DOI: $\underline{\text { http://dx.doi.org/10.1590/ }}$ s1413-82712005000200008.

25. Mondim EMC. Práticas educativas parentais e seus efeitos na criação dos filhos. Psicol Argum. 2008;26(54):233-44.

26. Cid MFB. Saúde mental de escolares: um estudo de prevalência e de fatores de risco e proteção [tese]. São Carlos (SP): Universidade Federal de São Carlos; 2011.

Recebido em: 3 de outubro de 2013. Aprovado em: 17 de março de 2014. 